\title{
Spontaneous Autoresuscitation in a Model of Respiratory Control
}

\author{
Casey O. Diekman ${ }^{1}$, Christopher G. Wilson ${ }^{2}$, and Peter J. Thomas ${ }^{3}$
}

\begin{abstract}
We introduce a closed-loop model of respiratory control incorporating a conductance-based central pattern generator (CPG), low-pass filtering of CPG output by the respiratory musculature, gas exchange in the lung, metabolic oxygen demand, and chemosensation. The CPG incorporates Butera, Rinzel and Smith (BRS)'s (1999) conditional pacemaker model. BRS model cells can support quiescent, bursting, or beating activity depending on the level of excitatory drive; we identify these activity modes with apnea (cessation of breathing), eupnea (normal breathing), and tachypnea (excessively rapid breathing). We demonstrate the coexistence of two dynamically stable behaviors in the closed-loop model, corresponding respectively to eupnea and tachypnea. The latter state represents a novel failure mode within a respiratory control model. In addition, the closed-loop system exhibits a form of autoresuscitation: conductances intrinsic to the BRS model buffer the CPG against brief episodes of hypoxia, steering the system away from catastrophic collapse as can occur with tachypnea.
\end{abstract}

\section{INTRODUCTION}

Rhythmic processes as diverse as heartbeat, respiration, locomotion, feeding, and cortical activity face a common challenge: adaptive regulation of central pattern generator (CPG) activity in response to fluctuating operational demands. Mathematical models of CPGs typically involve limit cycle (LC) dynamics. Models with LC dynamics are necessarily nonlinear [?], so the effects of regulatory feedback must be carefully taken into account. Moreover, the contribution of CPG dynamics to survival are mediated by the physical biomechanics of musculoskeletal effectors; considering a CPG circuit in isolation from biomechanics and sensory feedback can lead to incomplete understanding of a system [?].

Respiratory control in the mammalian brainstem provides a clinically significant case-in-point. The breathing rhythm is believed to originate in a small brainstem region, the preBötzinger complex (pBC) [17], containing c. 600 spontaneously rhythmic neurons [8]. The respiratory rhythm generated by the $\mathrm{pBC}$ is shaped and enhanced by complementary regions (e.g. RTN/pFRG, pons) [16]. Output from the pBC may be recorded on the hypoglossal XII nerve rootlet which drives the tongue to extend and open the airway during the

${ }^{1}$ C. O. Diekman is with the Mathematical Biosciences Institute, The Ohio State University, Columbus, OH 43210, USA cdiekman at mbi. osu. edu

${ }^{2} \mathrm{C}$. G. Wilson is with the Department of Pediatrics, Case Western Reserve University, Cleveland, $\mathrm{OH} 44106$, USA cgw5 at case.edu

${ }^{3} \mathrm{P}$. J. Thomas is with the Department of Mathematics, Case Western Reserve University, Cleveland, $\mathrm{OH}$ 44106, USA pjt 9 at case.edu This work was supported in part by NSF grants DMS-1010434 and EF1038677 to PJT, and by NSF Grant DMS-0931642 to the Mathematical Biosciences Institute, and by NIH Grant HL-62527 and HL-81622 to CGW. inspiratory phase of breathing. Butera, Rinzel and Smith (BRS) developed a model for spontaneously rhythmic cells in the $\mathrm{pBC}$, and studied the generation of respiratory-like rhythms in networks of up to 50 coupled cells in a heterogeneous all-to-all network [5], [6]. We focus here on the persistent sodium mediated conditional bursting cell model introduced in [5]. The BRS model cells show three distinct dynamical regimes as a function of tonic depolarizing drive representing exogenous input to the $\mathrm{pBC}$ circuit. Moderate drives lead to eupnea-like activity comprising regular bursts of electrical activity over a range of 6-60 bursts per minute, with intraburst spike frequencies of 2-50 spikes per second (cf. Fig. 2, top left panel). Insufficient drive leads to quiescence, corresponding physiologically to prolonged cessation of breathing or apnea [14]. Excessive drive, on the other hand, leads to a "beating" pattern, an uninterrupted train of single spikes in the range of 2-30 spikes per second (cf. Fig. 2, top right panel). Here we consider the possibility that the beating pattern produced by the BRS model, subject to large excitatory drive, may be a correlate of tachypnea, a pathological condition in which breathing is too rapid for effective gas exchange by the lungs [19].

\section{CLOSED-LOOP MODEL}

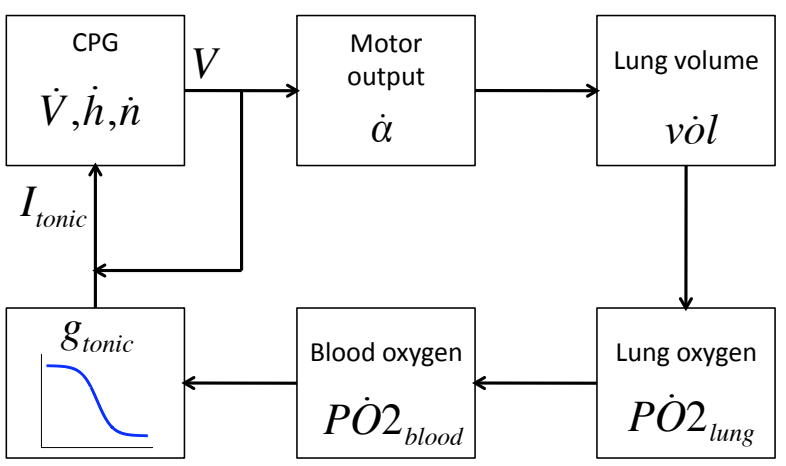

Fig. 1. Schematic of closed-loop control circuit. The model equations are given in the Appendix, except for the CPG gating variables $(h, n)$ which are as given in [5]. Parameter settings have been chosen to yield physiologically reasonable values for the model variables.

Respiratory homeostasis is maintained through multiple mechanisms, including chemosensory pathways sensitive to hypoxia [1] as well as to hypercapnia [11]. Insufficient excitatory drive is understood to be a possible factor in transient episodes of central apnea [14], defined as spontaneous interruption of breathing for 20 seconds or more in humans. A second, less well-studied failure mode can occur due to excessive positive feedback in a respiratory 
control system. In this scenario, excessive excitatory input can drive the system into the beating regime (tachypnea) leading to inefficient gas exchange and progressive blood $\mathrm{O}_{2}$ desaturation, thereby increasing the drive further. To investigate this phenomenon, we constructed a closed-loop respiratory control model incorporating chemosensory feedback (Fig. 1). Our CPG consists of a single Butera-RinzelSmith conditional pacemaker cell [5]; the pattern of activity is determined by the level of tonic excitatory input, $g_{\text {tonic }}$. For the parameters used $\left(\bar{g}_{N a P}=2.8 \mathrm{nS}, E_{L}=-65 \mathrm{mV}\right)$, when $g_{\text {tonic }} \lesssim 0.26$ the cell is quiescent; for $0.27 \lesssim g_{\text {tonic }} \lesssim 0.44$ it fires bursts of action potentials, and for $0.45 \lesssim g_{\text {tonic }}$ it fires a train of single spikes. The output of the CPG drives changes in lung volume through a linear motor pool variable $(\alpha(t))$. The lung and blood are included as single well-mixed compartments; oxygen transport and metabolic consumption depend linearly on oxygen tension (partial pressure of $\mathrm{O}_{2}$ in the lung and the blood, respectively). Chemosensation is mediated by a sigmoidal dependence of $g_{\text {tonic }}$ on PO2 blood . This highly simplified model omits many important aspects of respiratory control (see Discussion) but suffices to demonstrate stable eupnea, catastrophic self-reinforcing tachypnea, and autoresuscitation. Model equations are given in the Appendix.
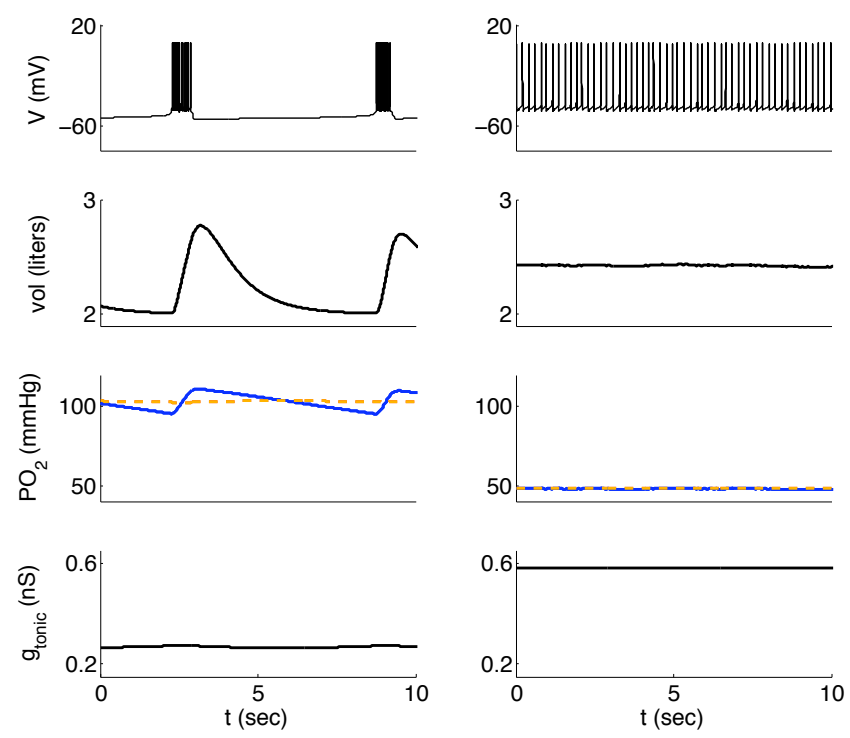

Fig. 2. Multistability in the closed-loop model. Simulations on the left and right differ only in their initial conditions. Traces from top to bottom: CPG voltage $(\mathrm{mV})$, lung volume (liters), lung (blue) and blood (orange) $\mathrm{PO}_{2}$ (mmHg), and drive to the CPG (nS). Left column: simulations in the bursting regime, corresponding to initial conditions from an open-loop simulation with $g_{\text {tonic }}=0.3 \mathrm{nS}$. Right column: simulations in the beating regime, corresponding to initial conditions from an open-loop simulation with $g_{\text {tonic }}=0.5 \mathrm{nS}$.

\section{TACHYPNEIC FAILURE MODE}

The closed-loop model (Fig. 1) has two dynamically stable long-term behaviors, corresponding to two of the three activity modes of the BRS equations [5]. Figure 2, left column, illustrates the operation of the circuit during normal breathing (eupnea). The tonic drive $\left(g_{\text {tonic }} \approx 0.3\right)$ supports a regular bursting pattern ( $V$, top panel) at a frequency that effectively drives the lungs (vol, second panel), leading to maintenance of $P O 2_{\text {blood }}$ close to normal operating levels $\left(100 \mathrm{mmHg}\right.$; PO2 $2_{\text {blood }}$, third panel). At this level of oxygenation, the chemosensory feedback provides a strong enough drive ( $g_{\text {tonic }} \approx 0.3$, bottom panel) to maintain activity. Small perturbations from the limit cycle representing normal operation quickly decay back to homeostasis. Figure 2, right column, illustrates tachypneic activity. If the tonic drive is above $0.45 \mathrm{nS}$ for an extended period of time, the CPG enters the beating regime ( $V$, top panel), producing motor output that is ineffective at driving changes in lung volume (vol, second panel), leading to desaturation of blood oxygen ( $P O 2_{\text {blood }}$, third panel). The resulting hypoxia elevates the chemosensory input, maintaining the CPG in the beating regime. This state is dynamically stable, in the sense that small perturbations from this behavior decay back to the same state. See Fig. 2 of [15] for an in vivo example of rapid, shallow breathing and oxygen desaturation in a human infant.

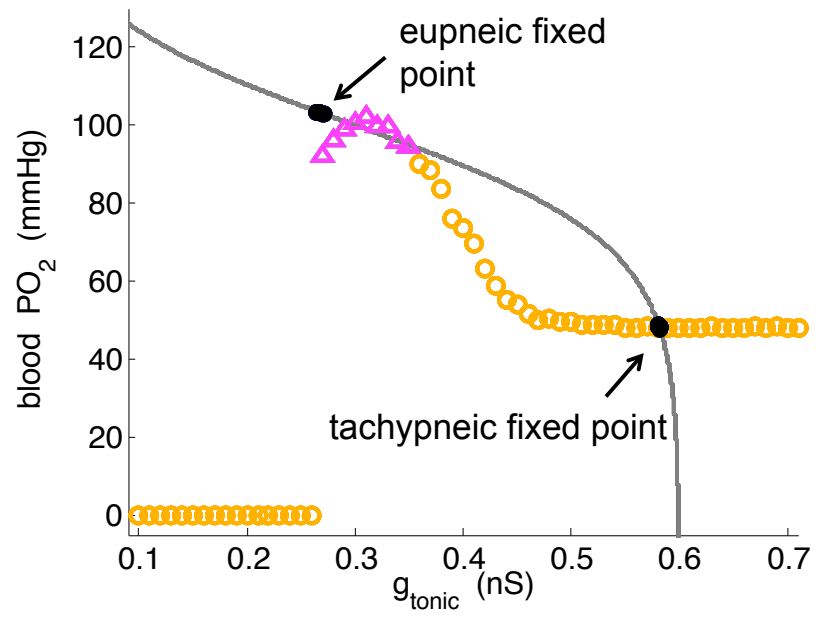

Fig. 3. Model steady-states. The open-loop model (orange circles and magenta triangles) has three distinct steady-state regimes corresponding to three qualitatively different behaviors of the CPG: quiescence $\left(g_{\text {tonic }} \lesssim 0.26\right)$, bursting $\left(0.27 \lesssim g_{\text {tonic }} \lesssim 0.44\right)$ and beating $\left(g_{\text {tonic }} \gtrsim 0.45\right)$. In contrast, the closed-loop model has just two distinct steady states: bursting and beating, corresponding to the eupneic and tachypneic fixed points (large black dots). The CPG is never quiescent in the closed-loop model, due to the increase in drive that occurs when the blood oxygen level starts to fall. Thus, insufficient drive is not a failure mode in this closed-loop control circuit. However, respiratory failure due to excessive drive does occur in this model. A decrease in blood oxygen and the subsequent increase in drive can cause a transition to the beating regime and inefficient gas exchange. The openloop blood oxygen values shown here are the average $P O 2_{\text {blood }}$ in the fifth minute of simulation starting with an initial $P O 2_{\text {blood }}$ of $100 \mathrm{mmHg}$. The closed-loop blood oxygen values are the average $\mathrm{PO}_{\text {blood }}$ in the fifth minute of simulation starting from the final state of the corresponding open-loop simulation.

Initial conditions leading to the eupneic and tachypneic states in Fig. 2 were obtained from open-loop simulations in which $g_{\text {tonic }}$ was initially fixed at 0.3 or $0.5 \mathrm{nS}$, respectively. Figure 3 shows $P O 2_{\text {blood }}$ obtained from an openloop configuration in which $g_{\text {tonic }}$ was clamped at values ranging from 0.1 to $0.7 \mathrm{nS}$. The gray curve shows the 
assumed sigmoidal dependence of the excitatory conductance $g_{\text {tonic }}$ on $P O 2_{\text {blood }}$; as blood oxygenation decreases the drive increases, but ultimately saturates near $g_{\text {tonic }} \lesssim 0.6 \mathrm{nS}$, in the beating regime. When $g_{\text {tonic }}$ is too low to support bursting behavior $\left(g_{\text {tonic }} \lesssim 0.26\right)$, there is no lung activity and eventually $P O 2_{\text {blood }}$ goes to zero. At intermediate levels of $g_{\text {tonic }}$, bursting activity is sustained and $P O 2_{\text {blood }}$ remains near normal levels. Higher levels of $g_{\text {tonic }}$ induce beating. Artificially clamping $g_{\text {tonic }}$ provides a means to explore the effects of short- and long-term imposition of hypoxia. When the drive is suppressed to the point of apnea, release from $g_{\text {tonic }}$-clamp immediately sends the CPG into an overexcited state producing a beating pattern that does not effectively restore oxygen balance. Similarly, when the drive is clamped above normal operating levels, the resulting relative hypoxia perpetuates the beating activity even after release of $g_{\text {tonic }}{ }^{-}$ clamp. Orange circles in Fig. 3 denote points along the open loop $P O 2_{\text {blood }} / g_{\text {tonic }}$ curve which, when used as initial conditions for the closed loop system, lead to persistent beating - the basin of attraction for tachypnea. The smaller set of magenta triangles denotes initial conditions in the basin of attraction for eupnea. The large black dots indicate the two asymptotically stable fixed points.

When $g_{\text {tonic }}$ is clamped to $g_{\text {tonic }} \approx 0.366 \mathrm{nS}$, the resulting bursting pattern is just sufficient to maintain $P O 2_{\text {blood }} \approx 93$ $\mathrm{mmHg}$. At this level, $g_{\text {tonic }} \approx 0.366$ when transduced through the sigmoid representing chemosensation. This value of $g_{\text {tonic }}$ represents an unstable fixed point of the dynamics. If $g_{\text {tonic }}$ is clamped $\lesssim 0.366 \mathrm{nS}$, the burst frequency is lower, with more spikes per burst; the gas exchange is more efficient, and $P O 2_{\text {blood }}$ is larger than $93 \mathrm{mmHg}$. When the $g_{\text {tonic }}$ clamp is released, bursting slows gradually, while becoming more efficient; $P O 2_{\text {blood }}$ rises, further reducing $g_{\text {tonic }}$ until the system rests at the eupneic stable fixed point. On the other hand, when $g_{\text {tonic }}$ is $\gtrsim 0.366 \mathrm{nS}$, the burst frequency is higher, with fewer spikes per burst; the gas exchange is less efficient, and $P O 2_{\text {blood }}$ falls below $93 \mathrm{mmHg}$. When the $g_{\text {tonic }}$ clamp is released, bursting accelerates, while becoming less efficient; $P O 2_{\text {blood }}$ declines, and the system transitions into the tachypneic fixed point.

\section{AUTORESUSCITATION}

Self-perpetuating tachypnea represents a catastrophic failure mode within the closed-loop respiratory control model presented here. However, the system shows unexpected resilience to transient imposition of hypoxia (Fig. 4). The system was allowed to generate a normal eupneic rhythm (corresponding to the upper fixed point in Fig. 3) until $t=60$ seconds, at which time $\mathrm{PO}_{\text {blood }}$ was artificially clamped ${ }^{1}$ to $80 \%$ of its normal level $(80 \mathrm{mmHg})$ for up to 10 seconds. Setting $P O 2_{\text {blood }}$ to $80 \mathrm{mmHg}$ increased the tonic drive to $g_{\text {tonic }} \approx 0.48$, well into the basin of attraction for tachypnea. However, the BRS model pacemaker did not respond by

\footnotetext{
${ }^{1}$ We chose this perturbation because $P O 2_{\text {blood }}$ is the slowest variable in our model. Thus, clamping it to a constant value is consistent with standard fast/slow analysis [?], and is equivalent to treating $g_{\text {tonic }}$ as a parameter in the open-loop configuration (Fig. 3).
}
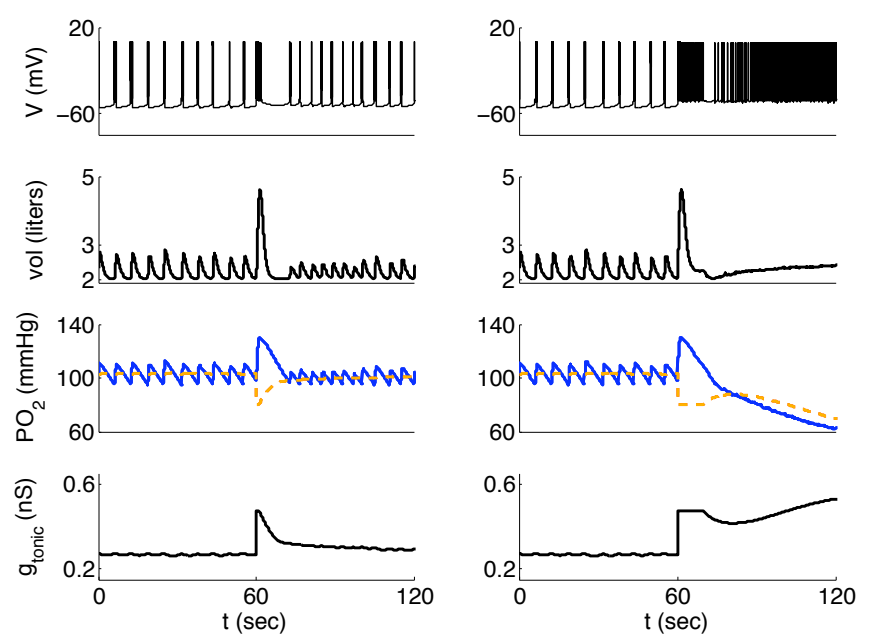

Fig. 4. Autoresuscitation in the closed-loop model. At $t=60 \mathrm{sec}, P O 2_{\text {blood }}$ was artificially reduced to $80 \mathrm{mmHg}$ and clamped there for $\tau$ seconds before being released. Left column: traces showing a barrage of spikes in response to a transient hypoxic challenge $(\tau=1)$ and the subsequent return to a eupneic breathing pattern. Right column: failure to recover from a hypoxic challenge of longer duration $(\tau=10)$.

immediately entering the stable beating pattern. Instead, it first produced a barrage of spikes longer and more intense than the preceding bursting pattern (Fig. 4, $V$, top panels). This transient barrage drove the lung compartment to expand more vigorously than normal (Fig. 4, vol, second panels). This additional lung expansion would lead to an increase in $P O 2_{\text {blood }}$, were $P O 2_{\text {blood }}$ not artificially clamped to 80 $\mathrm{mmHg}$. When the clamp was released after one second of simulated transient hypoxia, the barrage-induced lung expansion was still ongoing, and the blood oxygenation level recovered sufficiently to avoid spiraling into tachypnea (Fig. 4, left column). We refer to this phenomenon as spontaneous autoresuscitation. The transient response to hypoxic challenge buffers the system by steering it away from the overdriven state. This effect is an endogenous property of the BRS model conductances. For sufficiently prolonged hypoxia clamp, however, the transient terminates and the system experiences catastrophic tachypnea (Fig. 4, right column). The response of the system to imposed hypoxia of different durations and severities is illustrated in Fig. 5.

\section{DISCUSSION}

Negative consequences of positive feedback are not unfamiliar in excitable rhythmic biological systems. Excessive excitatory drive can lead to cessation of normal, functional oscillations. Examples include depolarization block in the classic Hodgkin-Huxley model [?] and ventricular tachycardia [18]. Closed-loop models incorporating chemosensation and gas exchange are important tools for developing clinically relevant predictions [2], [7], [9]. Previous models have incorporated respiratory CPG dynamics [3], [12] but to our knowledge a conductance-based CPG including fast spiking currents such as the BRS model has not previously been embedded in a respiratory control loop. Many important factors remain to be incorporated in future work: interaction 


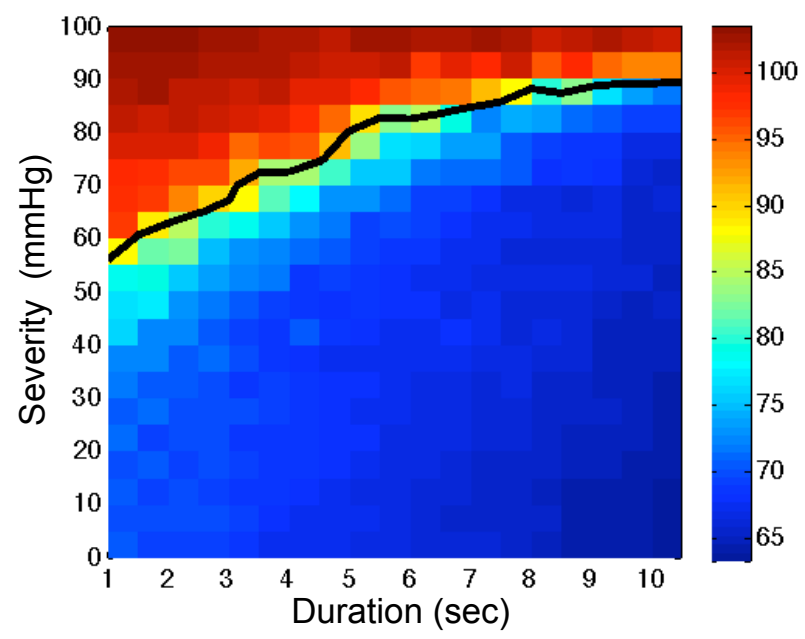

Fig. 5. Blood oxygen level 60 seconds after hypoxic challenges of varying severity and duration. Level curve (black) indicates $90 \mathrm{mmHg}$, and approximates a boundary above which the system recovers to eupneic breathing and below which the system descends into tachypnea.

of pBC-based rhythms with other brainstem areas [?] as well as cardiac rhythms [7]; biphasic response of infants to hypoxia [13], hypercapnia mediated chemosensory feedback [10], and the stabilizing effects present in larger networks of BRS cells [4], [6]. Nevertheless, our simplified model is sufficient to demonstrate a novel failure mode, self-sustaining tachypnea, as well as a surprising form of spontaneous autoresuscitation following transient hypoxic challenge. Both of these phenomena arise from conductances intrinsic to a well-studied respiratory central pattern generator model [5].

$$
\begin{aligned}
\text { APPENDIX } & \\
C \frac{d V}{d t} & =-I_{N a P}-I_{N a}-I_{K}-I_{L}-I_{\text {tonic }} \\
\frac{d \alpha}{d t} & =r[T](1-\alpha)-r \alpha \\
\frac{d}{d t}(v o l) & =-E_{1}\left(v o l-V o l_{0}\right)+E_{2} \alpha \\
\frac{d}{d t}\left(P O 2_{\text {lung }}\right) & =\left(\frac{1}{\text { vol }}\right)\left(\left(P O 2_{\text {ext }}-P O 2_{\text {lung }}\right) \times\right. \\
\frac{d}{d t}\left(P O 2_{\text {blood }}\right) & =B \times P O 2_{\text {lung }}-M \times P O 2_{\text {blood }} \\
g_{\text {tonic }} & =0.3\left(1-\tanh \left(\frac{d O 2_{\text {blood }}-100}{30}\right)\right)
\end{aligned}
$$

where $I_{\text {tonic }}=g_{\text {tonic }}\left(V-E_{\text {syne }}\right),[T]=T_{\text {max }} /(1+\exp (-(V-$ $\left.\left.\left.V_{T}\right) / K_{p}\right)\right), T_{\max }=1, r=0.001, V_{T}=2, K_{p}=5, E_{1}=$ $0.0025, E_{2}=0.4$, Vol $_{0}=2, P O 2_{\text {ext }}=159.6, A=6 \times 10^{-5}$ and $B=M=7 \times 10^{-5} \cdot[x]_{+}$denotes $\max (x, 0)$. The expressions for $C, I_{N a P}, I_{N a}, I_{K}$, and $I_{L}$ are as given in [5].

\section{ACKNOWLEDGMENTS}

We thank G. Longobardo, C. Evangelisti, J. Rubin, D. Calvetti, H. Chiel, and E. Somersalo for helpful discussions.

\section{REFERENCES}

[1] S Andronikou, M Shirahata, A Mokashi, and S Lahiri. Carotid body chemoreceptor and ventilatory responses to sustained hypoxia and hypercapnia in the cat. Respir Physiol, 72(3):361-74, Jun 1988.

[2] Musa H Asyali, Richard B Berry, and Michael C K Khoo. Assessment of closed-loop ventilatory stability in obstructive sleep apnea. IEEE Trans Biomed Eng, 49(3):206-16, Mar 2002.

[3] Alona Ben-Tal and Jeffrey C Smith. A model for control of breathing in mammals: coupling neural dynamics to peripheral gas exchange and transport. J Theor Biol, 251(3):480-97, Apr 2008.

[4] J Best, A Borisyuk, J Rubin, D Terman, and M Wechselberger. The dynamic range of bursting in a model respiratory pacemaker network. SIAM J Appl Dyn Sys, 4:1107-1139, 2005.

[5] Robert J. Butera Jr., John Rinzel, and Jeffrey C. Smith. Models of Respiratory Rhythm Generation in the preBötzinger Complex. I. Bursting Pacemaker Neurons. J Neurophysiol, 82(1):382-397, 1999.

[6] Robert J. Butera Jr., John Rinzel, and Jeffrey C. Smith. Models of Respiratory Rhythm Generation in the preBötzinger Complex. II. Populations of Coupled Pacemaker Neurons. J Neurophysiol, 82(1):398-415, 1999.

[7] Limei Cheng, Olga Ivanova, Hsing-Hua Fan, and Michael C K Khoo. An integrative model of respiratory and cardiovascular control in sleepdisordered breathing. Respir Physiol Neurobiol, 174(1-2):4-28, Nov 2010.

[8] John A Hayes and Christopher A Del Negro. Neurokinin receptorexpressing pre-Bötzinger complex neurons in neonatal mice studied in vitro. J Neurophysiol, 97(6):4215-4224, Jun 2007.

[9] M C Khoo and M E Benser. "Optimal" application of ventilatory assist in Cheyne-Stokes respiration: a simulation study. Conf Proc IEEE Eng Med Biol Soc, 6:5832-5, 2005.

[10] M C Khoo, R E Kronauer, K P Strohl, and A S Slutsky. Factors inducing periodic breathing in humans: a general model. $J$ Appl Physiol, 53(3):644-59, Sep 1982.

[11] M C Khoo and V Z Marmarelis. Estimation of peripheral chemoreflex gain from spontaneous sigh responses. Ann Biomed Eng, 17(6):557$70,1989$.

[12] Guy Longobardo, Carlo J Evangelisti, and Neil S Cherniack. Introduction of respiratory pattern generators into models of respiratory control. Respir Physiol Neurobiol, 148(3):285-301, Oct 2005.

[13] I R Moss. Respiratory responses to single and episodic hypoxia during development: mechanisms of adaptation. Respir Physiol, 121(23):185-97, Jul 2000

[14] H Rigatto and J P Brady. Periodic breathing and apnea in preterm infants. I. Evidence for hypoventilation possibly due to central respiratory depression. Pediatrics, 50(2):202-18, Aug 1972.

[15] H Rigatto and J P Brady. Periodic breathing and apnea in preterm infants. II. Hypoxia as a primary event. Pediatrics, 50(2):219-28, Aug 1972.

[16] Jonathan E Rubin, Natalia A Shevtsova, G Bard Ermentrout, Jeffrey C Smith, and Ilya A Rybak. Multiple rhythmic states in a model of the respiratory central pattern generator. J Neurophysiol, 101(4):2146-65, Apr 2009.

[17] JC Smith, HH Ellenberger, K Ballanyi, DW Richter, and JL Feldman. Pre-Bötzinger complex: a brainstem region that may generate respiratory rhythm in mammals. Science, 254(5032):726-729, 1991.

[18] N E Torres and R D White. Current concepts in cardiopulmonary resuscitation. J Cardiothorac Vasc Anesth, 11(3):391-407, May 1997.

[19] Murat Yurdakok and Eren Ozek. Transient tachypnea of the newborn: The treatment strategies. Curr Pharm Des, Feb 2012 